\title{
SyNumSeS: A Python Package for Numerical Simulation of Semiconductor Devices
}

\author{
Peter Abele ${ }^{1 *}$, Michael Schäfer $^{2}$ \\ ${ }^{1}$ HTWG Konstanz - University of Applied Sciences, 78462 Konstanz, Alfred-Wachtel-Str. 8, Germany \\ *pabele@htwg-konstanz.de \\ ${ }^{2}$ CADwalk GmbH \& Co. KG, Joseph-von-Sontheimer-Str. 3, 89604 Allmendingen, Germany
}

SNE 31(2), 2021, 81-85, DOI: 10.11128/sne.31.sw.10566 Received: May 26, 2021 (Selected ASIM WS GMMS/STS 2021 Postconf. Publ.), Accepted: June 5, 2021

SNE - Simulation Notes Europe, ARGESIM Publisher Vienna, ISSN Print 2305-9974, Online 2306-0271, www.sne-journal.org

\begin{abstract}
SyNumSeS is a Python package for numerical simulation of semiconductor devices. It uses the Scharfetter-Gummel discretization for solving the one dimensional Van Roosbroeck system which describes the free electron and hole transport by the drift-diffusion model. As boundary conditions voltages can be applied to Ohmic contacts. It is suited for the simulation of pndiodes, MOS-diodes, LEDs (hetero junction), solar cells, and (hetero) bipolar transistors.
\end{abstract}

\section{Introduction}

The development of modern semiconductor devices is accompanied by numerical simulations. This results in a better understanding of the device as physical quantities like electrical fields or current densities can be visualized and related to material parameters or the geometry of the device. This way time consuming experiments can be reduced saving resources, time, and money. In teaching semiconductor devices the visualization of what is happening inside the device is very helpful for the students. So they can get a better link between the learned equations and what do they mean for the electrical properties of the device. Presenting animations showing the output characteristics and for example the hole and electron density in the device for the different bias regions is often better then thousands of words. This was the motivation for programming a simple to use and fast Python package for numerical simulations of semiconductor devices.

This package is used for the lecture 'Electron Devices' to generate diagrams and animations. In the mas- ter's course 'Microfabrication Techniques' the students use the package within a lab for the simulations of pndiodes, LEDs, solar-cells and SiGe hetero bipolar transistors.

\section{Solving the Basic Semiconductor Equations}

The Van Roosbroeck equations [1] are used as the basic semiconductor equations. For one dimension they are:

$$
\begin{array}{r}
-\frac{\partial}{\partial x}\left(\varepsilon_{0} \varepsilon_{r} \frac{\partial}{\partial x} \Psi\right)=q_{\mathrm{e}} \cdot(C+p-n) \\
\frac{\partial}{\partial t} n=\frac{1}{q_{\mathrm{e}}} \cdot \frac{\partial}{\partial x} j_{\mathrm{n}}+(G-R), \\
\frac{\partial}{\partial t} p=-\frac{1}{q_{\mathrm{e}}} \cdot \frac{\partial}{\partial x} j_{\mathrm{p}}+(G-R) .
\end{array}
$$

These are three nonlinear ordinary differential equations for three unknowns, the potential $\Psi$, the free electron density $n$, and the free hole density $p$. ( $C$ is the doping concentration, $G$ and $R$ are the generation and recombination rates, respectively, $\varepsilon_{0}$ is the dielectric constant, and $\varepsilon_{r}$ is the relative permittivity.) These equations can be solved by using partial differential equation (PDE) solvers like FiPy[2]. As for semiconductor devices high electrical fields and extreme concentration gradients for electrons and holes exist especially at pnor hetero-junctions using a general PDE solver results in significant deviation from the exact solution. This can be partly compensated by reducing the grid size but at the expense of simulation time and even convergence issues.

By using the Scharfetter Gummel scheme [3][4] for the electron and hole current densities this issue can be relaxed. 
Additionally in the equations (1) the unknowns $n$ and $p$ are replaced by the quasi Fermi levels for electrons $\Phi_{\mathrm{n}}$ and holes $\Phi_{\mathrm{p}}$, respectively using the flowing relations:

$$
n=N_{\mathrm{C}} \cdot \exp \left(\frac{X-\Phi_{\mathrm{n}}+\Psi}{U_{\mathrm{t}}}\right)
$$

and

$$
p=N_{\mathrm{V}} \cdot \exp \left(\frac{-X-E_{\mathrm{g}}+\Phi_{\mathrm{p}}-\Psi}{U_{\mathrm{t}}}\right)
$$

With $X^{1}$ being the electron affinity, $E_{\mathrm{g}}$ the band gap, and $U_{\mathrm{t}}=\frac{k_{\mathrm{B}} T}{q_{\mathrm{e}}}\left(k_{\mathrm{B}}\right.$ Boltzmann constant, $T$ absolute temperature, and $q_{\mathrm{e}}$ elementary charge). $N_{\mathrm{C}}$ and $N_{\mathrm{V}}$ are the effective density of states for the conduction band and the valence band, respectively.

For solving this PDEs the finite volume method for one dimension is used. The electron and hole current densities between two adjacent cells are expressed by:

$$
\begin{aligned}
& j_{\mathrm{n} ; \mathrm{k}, \mathrm{l}}= \\
& -\frac{q_{\mathrm{e}} U_{\mathrm{t}}}{\Delta x} \cdot \frac{\mu_{\mathrm{n}, \mathrm{k}}+\mu_{\mathrm{n}, \mathrm{l}}}{2} \cdot \frac{N_{\mathrm{C}, \mathrm{k}}+N_{\mathrm{C}, \mathrm{l}}}{2} \cdot \exp \left(\frac{X_{\mathrm{k}}+X_{\mathrm{l}}}{2}\right) \cdot[ \\
& B\left(-\frac{\Psi_{1}-\Psi_{\mathrm{k}}}{U_{\mathrm{t}}}\right) \cdot \exp \left(\frac{\Psi_{\mathrm{k}}-\Phi_{\mathrm{n}, \mathrm{k}}}{U_{\mathrm{t}}}\right) \\
& -B\left(\frac{\Psi_{1}-\Psi_{\mathrm{k}}}{U_{\mathrm{t}}}\right) \cdot \exp \left(\frac{\Psi_{\mathrm{l}}-\Phi_{\mathrm{n}, \mathrm{l}}}{U_{\mathrm{t}}}\right) \\
& j_{\mathrm{p} ; \mathrm{k}, \mathrm{l}}= \\
& -\frac{q_{\mathrm{e}} U_{\mathrm{t}}}{\Delta x} \cdot \frac{\mu_{\mathrm{p}, \mathrm{k}}+\mu_{\mathrm{p}, \mathrm{l}}}{2} \cdot \frac{N_{\mathrm{V}, \mathrm{k}}+N_{\mathrm{V}, 1}}{2} \cdot \exp \left(\frac{X_{\mathrm{k}}+E_{\mathrm{g}, \mathrm{k}}+X_{\mathrm{l}}+E_{\mathrm{g}, 1}}{2}\right) \cdot[ \\
& B\left(-\frac{\Psi_{1}-\Psi_{\mathrm{k}}}{U_{\mathrm{t}}}\right) \cdot \exp \left(\frac{\Phi_{\mathrm{p}, \mathrm{k}}-\Psi_{\mathrm{k}}}{U_{\mathrm{t}}}\right) \\
& -B\left(\frac{\Psi_{1}-\Psi_{\mathrm{k}}}{U_{\mathrm{t}}}\right) \cdot \exp \left(\frac{\Phi_{\mathrm{p}, 1}-\Psi_{1}}{U_{\mathrm{t}}}\right)
\end{aligned}
$$

With $k$ and $l$ being indices of adjacent cells, $\mu_{\mathrm{n}}$ and $\mu_{\mathrm{p}}$ are the mobilities of electrons and holes, respectively, and $\Delta x$ is the cell distance. $B(x)$ is the Bernoulli function:

$$
B(x)=\frac{x}{\exp (x)-1} .
$$

Compared to [4] the equations (4) and (5) are slightly modified by the terms containing the electron affinity $X$ to account for hetero junctions.

Care must be taken for the Bernoulli function in the case of low values for the argument as this results in a low value divided by an other low value. Therefore the Bernoulli function is approximated by a polynomial of degree 1 for low values of the argument:

$$
B(x)=1-\frac{x}{2} \text {. }
$$

\section{Boundary Condition}

On the very left and right of the one dimensional device boundary conditions for Ohmic contacts are set and different voltages can be applied to both sides. This is done by adding one additional cell on the left and right with the quasi Fermi levels and potential set to [5]:

$$
\begin{gathered}
\Phi_{\mathrm{n}}=V_{\mathrm{A}} \\
\Phi_{\mathrm{p}}=V_{\mathrm{A}} \\
\Psi=-X-\frac{E_{\mathrm{g}}+U_{\mathrm{t}} \log \left(\frac{N_{\mathrm{C}}}{N_{\mathrm{V}}}\right)}{2}+U_{\mathrm{t}} \operatorname{arcsinh}\left(\frac{C}{2 n_{\mathrm{i}}}\right)+V_{\mathrm{A}}
\end{gathered}
$$

With $V_{\mathrm{A}}$ the applied voltage and $n_{\mathrm{i}}$ the intrinsic carrier density. For the simulation of bipolar transistors an additional contact between the outer contacts is needed. For this contact dependent on the doping, p- or n-type, only the potential $\Psi$ and one of the quasi Fermi levels $\Phi_{\mathrm{p}}$ or $\Phi_{\mathrm{n}}$ are set, respectively.

\section{Solving the Semiconductor Equations}

The discretization of the equations (1) using the equations (2)-(5) results in a nonlinear equation system for the unknowns $\Psi, \Phi_{\mathrm{n}}$, and $\Phi_{\mathrm{p}}$ for every cell. To solve this nonlinear equation system Newton's method is used. For building the Jacobian matrix the equations are symbolically derived using the Python package sympy. The code for solving these equations is automatically generated by a Python script.

\footnotetext{
${ }^{1}$ For ease of use the units of $X, E_{\mathrm{g}}, \Phi_{\mathrm{n}}$, and $\Phi_{\mathrm{p}}$ are $\mathrm{V}$ and not $\mathrm{eV}$ !
} 


\section{Simulation Results}

To demonstrate the wide use of this package simulation results for three different semiconductor devices are presented.

\subsection{Simulation results of an InGaN LED}

For the simulation of an $\mathrm{LED}^{2}$ the InGaN material system is used. The well, where the light is generated, consists of $\operatorname{In}_{0.3} \mathrm{Ga}_{0.7} \mathrm{~N}$ with a band gap of about $E_{\mathrm{g}}=$ $2.0 \mathrm{eV}$ and has a thickness of $100 \mathrm{~nm}$. The surrounding material is $\mathrm{GaN}$ with $E_{\mathrm{g}}=3.4 \mathrm{eV}$. The $\mathrm{In}_{0.3} \mathrm{Ga}_{0.7} \mathrm{~N}$ well is not doped while the surrounding $\mathrm{GaN}$ to the left is p-doped and to the right is n-doped both with a concentration of $N_{\mathrm{A}}=N_{\mathrm{D}}=2 \cdot 10^{22} \mathrm{~m}^{-3}$.

Fig. 1 shows the resulting band diagram with no externally applied voltage.

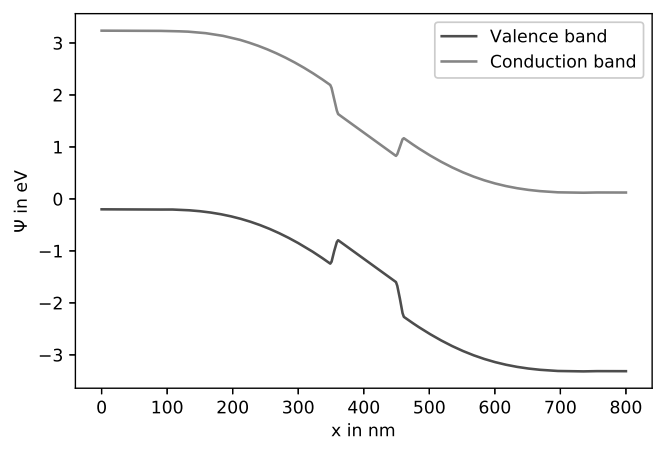

Figure 1: Band diagram of InGaN-LED with no externally applied voltage.

In the center the $\operatorname{In}_{0.3} \mathrm{Ga}_{0.7} \mathrm{~N}$ well can be clearly seen and the band bending respectively build in voltage $\Phi_{\mathrm{bi}}$ due to the doping concentrations. Simulating the output characteristics shows a diode threshold voltage of about $V_{\text {th }}=2.8 \mathrm{~V}$, see Fig. 2 .

The nearly linear increase of the current density beyond $V_{\text {th }}$ can be attributed to the low doping level of the $\mathrm{GaN}$ resulting in a relatively high Ohmic resistance.

The recombination rate is considered by:

$$
R=C_{\mathrm{rad}} \cdot\left(n p-n_{\mathrm{i}}^{2}\right) .
$$

As the free electrons and holes accumulate at the well we get in this region the highest recombination rate, see Fig. 3.

\footnotetext{
${ }^{2}$ Light emitting diode
}

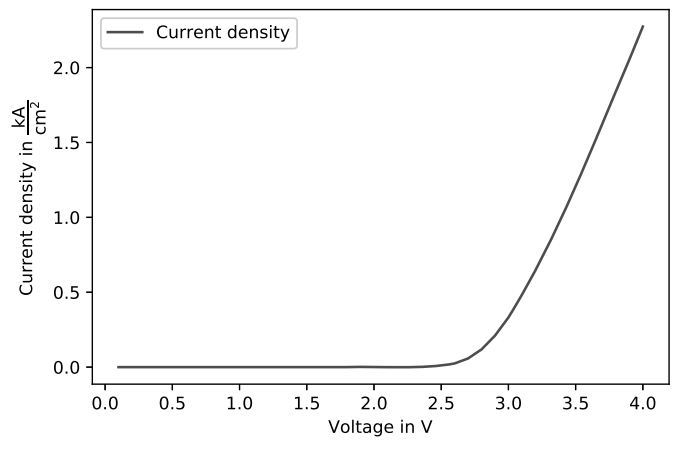

Figure 2: LED characteristic.

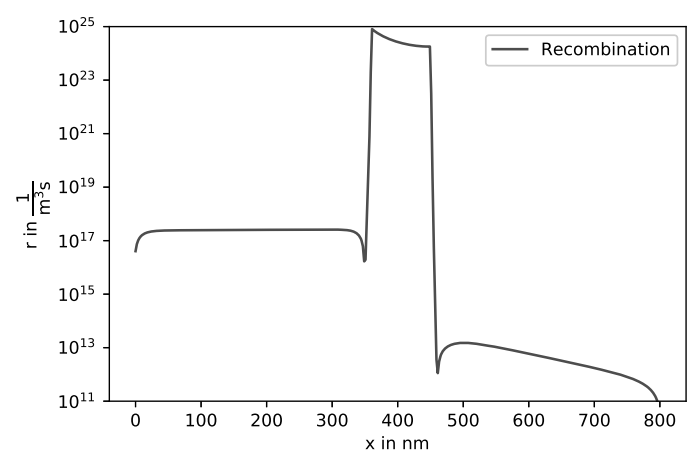

Figure 3: Recombination rate along the LED.

\subsection{Simulation results of solar cells}

In the following silicon solar cells are simulated. The thickness of the silicon is $d=1 \mu \mathrm{m}$. The first $40 \mathrm{~nm}$ from the surface are p-doped with $N_{\mathrm{A}}=1.0 \cdot 10^{24} \mathrm{~m}^{-3}$. The rest of the silicon is n-doped with different concentrations. The intensity of the incident light drops exponentially while penetrating through the silicon and generate free electrons and holes.

In the following the influence of the different ndoping concentrations, $N_{\mathrm{D}}=1.25 \cdot 10^{21} \mathrm{~m}^{-3}, N_{\mathrm{D}}=1.0$. $10^{22} \mathrm{~m}^{-3}$, and $N_{\mathrm{D}}=8.0 \cdot 10^{22} \mathrm{~m}^{-3}$ is shown. The intensity of the incident light is kept constant.

The doping level has two effects, first on the build in voltage $\Phi_{\mathrm{bi}}$, which increases with increasing doping level, and second the extension of the space charge region which decreases with increasing doping level. A higher build in voltage results in a higher idle voltage, this can be seen in Fig. 4. But on the other side with a reduction of the extension of the space charge region the short circuit current drops. 


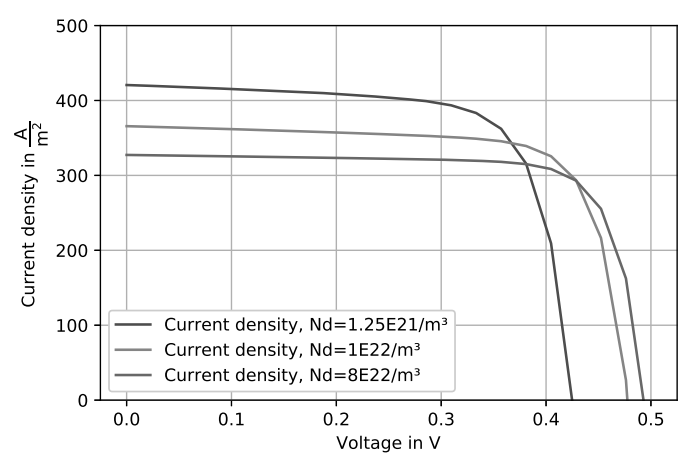

Figure 4: Solar cell output characteristic for different n-doping concentrations.

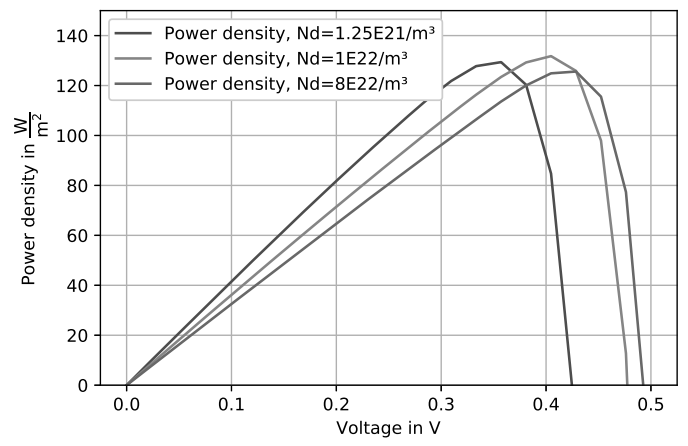

Figure 5: Output power density of differently doped solar cells.

For optimizing the doping concentration in Fig. 5 the power density dependent on the applied voltage is shown. The maximum power point is for the doping levels of $N_{\mathrm{D}}=1.25 \cdot 10^{21} \mathrm{~m}^{-3}$ and $N_{\mathrm{D}}=1.0 \cdot 10^{22} \mathrm{~m}^{-3}$ about the same. For the higher doping level it is about $5 \%$ lower.

\subsection{Simulation results of a Si bipolar transistor}

The following shows simulation results of a npn bipolar transistor. The device has an extension of $1.2 \mu \mathrm{m}$. The first $400 \mathrm{~nm}$ build the emitter which is $\mathrm{n}$-doped with $N_{\mathrm{D}, \mathrm{E}}=1.2 \cdot 10^{24} \mathrm{~m}^{-3}$. The next $400 \mathrm{~nm}$ build the base which is p-doped with $N_{\mathrm{A}, \mathrm{B}}=4.0 \cdot 10^{22} \mathrm{~m}^{-3}$. The last $400 \mathrm{~nm}$ build the collector which is n-doped with $N_{\mathrm{D}, \mathrm{C}}=2.0 \cdot 10^{22} \mathrm{~m}^{-3}$.
For this simulation the base contact is located at $x=$ $650 \mathrm{~nm}$. As SyNumSeS can only solve devices for one dimension, the boundary condition at the base contact (p-doped) only fixes the potential $\Psi$ and the quasi Fermi level for the holes $\Phi_{\mathrm{p}}$. The quasi Fermi level for the electrons $\Phi_{\mathrm{n}}$ at this point is calculated by the nonlinear equation system. Thereby the electrons can pass the base contact.

In a first step the base emitter voltage is ramped from $V_{\mathrm{BE}}=0 \mathrm{~V}$ to $V_{\mathrm{BE}}=0.6 \mathrm{~V}$ in steps of $0.1 \mathrm{~V}$. Then the collector emitter voltage is ramped to $V_{\mathrm{CE}}=3.0 \mathrm{~V}$ in steps of $50 \mathrm{mV}$. This results in the output characteristics and is show in Fig. 6.

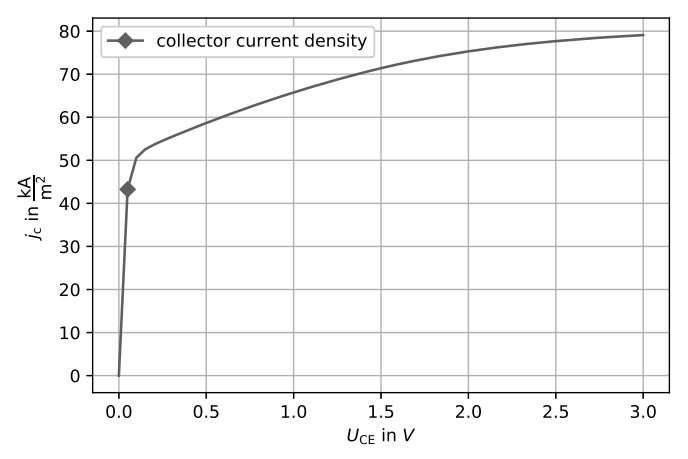

Figure 6: Output characteristics of a Si bipolar transistor for

$$
V_{\mathrm{BE}}=0.6 \mathrm{~V} \text {. }
$$

The simulated output characteristics shows the saturation region up to about $V_{\mathrm{CE}}=100 \mathrm{mV}$. The following slope can be attributed to the Early effect.

To get a better understanding for the saturation region, we can have a look at the electron and hole current density across the device at $V_{\mathrm{CE}}=50 \mathrm{mV}$ and $V_{\mathrm{BE}}=0.6 \mathrm{~V}$, see Fig. 7 .

It can be clearly seen that electrons are not only injected from the emitter into the base, but also from the collector into the base $\left(400 \mathrm{~nm}<x_{\text {Base }}<800 \mathrm{~nm}\right)$. So the gradient of the electron density is low, resulting in a low collector current caused by diffusion.

\section{Summary}

The Python package SyNumSeS has proven its capability for the simulation of various semiconductor devices. As these devices are also discussed in bachelor and master courses the package is well suited to support the lecture by visualizing physical quantities in the 


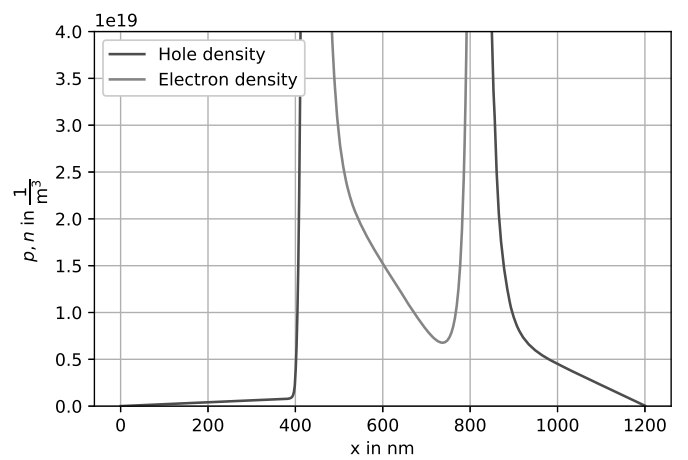

Figure 7: Electron and hole density across the bipolar transistor at saturation for $V_{\mathrm{CE}}=50 \mathrm{mV}$ and $V_{\mathrm{BE}}=0.6 \mathrm{~V}$.

device for different bias regions. Due to its fast simulation times it is also appropriate for lab courses where the students can investigate the influence of process and material parameters on the performance of a device.

This Python package can be installed by:

pip install synumses-pkg-pabele

and cloned from

https://github.com/pabele/synumses

with several examples using the JupyterLab note-

book.

\section{References}

[1] Van Roosbroeck W. Theory of the Flow of Electrons and Holes in Germanium and Other Semiconductors. Bell System Technical Journal. 1950;29(4):560-607.

[2] Guyer JE, Wheeler D, Warren JA. FiPy: Partial Differential Equations with Python. Computing in Science \& Engineering. 2009;11(3):6-15.

[3] Scharfetter DL, Gummel HK. Large-signal analysis of a silicon Read diode oscillator. IEEE Transactions on Electron Devices. 1969;16(1):64-77.

[4] Farrell P, Rotundo N, Doan DH, Kantner M, Fuhrmann J, Koprucki T. Drift-Diffusion Models. In: Handbook of Optoelectronic Device Modeling and Simulation, edited by Piprek J, pp. 733-772. London, New York: CRC Press. 2017;

[5] Selberherr S. Analysis and Simulation of Semiconductor Devices. Wien, New York: Springer. 1984. 